\title{
Neue Wege der Esskultur: Zwischen Askese, Völlerei und Binge-Eating
}

\author{
Gaby-Fleur Böl ${ }^{1}$
}

Published online: 1 February 2017

(C) Bundesamt für Verbraucherschutz und Lebensmittelsicherheit (BVL) 2017

Haben Sie in letzter Zeit mal Freunde zum Essen eingeladen? Falls ja, gibt es eine gewisse Wahrscheinlichkeit, dass die üblichen Spaßbremsen dabei waren, dies und das an die Seite des Tellers aussortierten und spätestens zum Kaffee Sojamilch haben wollten, weil sie „von Kuhmilch wegen der Laktose immer Magendrücken bekommen“. Dann doch lieber gemeinsam essen gehen, da kann sich jeder im Restaurant das aussuchen, was ihm schmeckt und was er verträgt. Lebensmittelallergie, Unverträglichkeit oder schlicht eine Modeerscheinung? Heutzutage isst man bestimmte Dinge einfach nicht, wenn man etwas auf sich hält. Verzicht ist das neue „Mehr“: Veganer, Vegetarier, Pescetarier, Freeganer, Flexitarier, Frutarier, Steinzeitler und Rohköstler bevölkern die Welt, wo es früher nur eine Einteilung in „bio“ und „konventionell“ gab. Zugehörigkeit zu einer bestimmten Peergroup macht frei und schafft moralische Überlegenheit gegenüber jenen, die doch tatsächlich noch alles essen. Im Supermarkt wird der Einkauf dank dieser Komplexitätsreduktion deutlich erleichtert, wenn man nicht in der enormen Vielfalt des Angebotes versinkt, sondern gezielt nach Produkten „frei von..." sucht: frei von Fett oder Gluten klingt ja genauso gut wie frei von Zusatz- oder Konservierungsstoffen, und die sind ja schon per se giftig, so denkt man. Händler können diese Produkte sogar

Gaby-Fleur Böl

gaby-fleur.boel@bfr.bund.de

1 Abteilung Risikokommunikation, Bundesinstitut für Risikobewertung, Max-Dohrn-Strasse 8-10, 10589 Berlin, Germany teuer verkaufen, da Kunden sie Befragungen zufolge als hochwertiger einstufen.

Gab es früher noch bemitleidenswerte Ecken für Diabetiker, bei denen sämtliche Lebensmittel - heute wissenschaftlich völlig überholt - Fruktose statt Glukose enthielten, ist es heutzutage eher schick, besondere, in irgendeiner Art reduzierte Lebensmittel auszusuchen. So jemand macht sich eben Gedanken um seine Ernährung und stopft nicht alles gedankenlos in sich hinein wie ein Omnivor. Statt Weizen isst man eben eher Quinoa oder Amaranth, auch wenn man gar nicht an Zöliakie leidet und somit konsequent auf Gluten verzichten muss. Heutzutage definiert man sich also weniger über das, was man isst, als über das, was man nicht isst. Wird der Hype zur Obsession, kann das krankhaft werden und nennt sich dann Orthorexie - der krampfhafte Versuch, den ganzen Tag nur gesundes und richtiges Essen zu finden. Rund ein Drittel der deutschen Bevölkerung meint aktuellen Umfragen zufolge an Lebensmittelunverträglichkeiten oder Allergien zu leiden. Von den restlichen zwei Dritteln verzichten etwa $80 \%$ auf bestimmte Lebensmittel, um sich etwas Gutes zu tun. Angesichts weltweit zunehmender Übergewichtigkeit in den Industriestaaten klingt der Ansatz des „Clean Eatings“ zunächst gut: Statt wie früher schlicht Kalorien zu zählen, verbirgt sich hier ein ganzheitliches Modell - mehr gute Fette, weniger Zucker, Salz und Alkohol; keine Farb-, Konservierungs-, Aroma- oder Süßstoffe entbehrt jedoch jeglicher wissenschaftlichen Grundlage. Dennoch sind sowohl Übergewicht als auch Magersucht meist Folgen gestörten Essverhaltens. Es stellt sich die Frage, wie es dazu kommen konnte - ein Phänomen, 
das jeder neunte Mensch der Weltbevölkerung gar nicht kennen kann, da er oder sie nicht genug zum Essen hat. Die meisten davon (98\%) leben in Entwicklungsländern in Afrika, Asien und der Pazifikregion. Noch heute sterben mehr Menschen jährlich an Hunger als an AIDS, Malaria und Tuberkulose - ein lösbares Problem.

Essen soll schmecken und gesund sein - zumindest hier sind die Deutschen nach Angaben des Ernährungsreports 2017 der Bundesregierung mit über 90\% der 1.000 Befragten einer Meinung. Mehr als die Hälfte der Deutschen essen am liebsten Fleisch, während nur jeder Fünfte Gemüse als Leibgericht nennt. Der Anteil, der täglich oder gelegentlich selbst zu Hause kocht, sinkt kontinuierlich von Jahr zu Jahr und liegt derzeit bei knapp $40 \%$ der Bundesbürger. Als Einkaufskriterien gelten in Deutschland in absteigender Reihenfolge unverändert Geschmack, Regionalität bzw. der Preis von Lebensmitteln, wobei hierbei Einkäufe im Supermarkt mit $62 \%$ weit vorne liegen. Während eine zunehmende Ernährungselite hedonistisch genießt und sich Gedanken um fairen Handel und Nachhaltigkeit machen kann, ist dies den meisten Menschen aufgrund ökonomischer Zwänge verwehrt. Dennoch wollen alle gesunde, preiswerte und sichere Lebensmittel kaufen, was in der heutigen industrialisierten Welt dank moderner Anbau-, Ernte-, Lager- und Transportbedingungen mehr denn je möglich ist.

Erdbeeren im Winter, Orangen im Sommer, Spargel zu jeder Jahreszeit? Die ständige Verfügbarkeit einer schier unendlichen Auswahl an Lebensmitteln macht uns das Leben paradoxerweise schwer. Während die menschliche Neugier uns antreibt, immer neue Ernährungstrends - wie beispielsweise auch das Essen von Insekten als proteinreiche Nahrungsquelle - zu testen, bremst uns oft die "German Angst" vor allem, was vielleicht doch schädlich für unseren Körper sein könnte. Gefühlte, also nicht wissenschaftlich begründete Risiken gehören zum gesellschaftlichen Leben und prägen das Verhalten der Menschen im Alltag. Wem kann man dann verdenken, dass Essen per se emotional aufgeladen ist und anscheinend eher eine spirituelle Erfahrung denn Nahrungs-aufnahme bedeutet?

Während Kochsendungen in TV-Shows boomen, sitzen die Deutschen mit einer Plastikschale davor und essen Fast Food. Deutschland kennt Fast Food Restaurants seit der Eröffnung der ersten Filiale von McDonald's im Jahr 1970 in München. Übergewicht versus Essstörung - manch einer wünscht sich dann doch die regelmäßige gemeinsame Mahlzeit unserer Kindertage mit der Familie zurück.

Digital Natives von heute können teilweise nur noch schwerlich ein Schaf von einer Ziege unterscheiden, vom kindlichen Glauben an die Existenz von lila Kühen ganz zu schweigen. Romantisierende Werbung wie die vom Mönch, der genussvoll und mit Bedacht den Käse rührt oder von der Kuh, die allein auf einer saftigen Weide hoch oben auf der Alm grast, machen sich vielleicht gut für Werbezwecke, konterkarieren jedoch den Anspruch an Glaubhaftigkeit und befördern so die Skepsis gegenüber Lebensmitteln unserer Tage nur noch mehr. Transparenz wie bei gläsernen Schlachthöfen wäre wünschenswert - und das ist, entgegen der Befürchtung besorgter Eltern, auch sensiblen Kinderseelen zumutbar. Nur dann, wenn man als vertrauensbildende Maßnahme sehen kann, wie Fleisch oder eben auch andere Lebensmittel tatsächlich erzeugt werden, lassen sich fundierte Entscheidungen treffen, was man tatsächlich essen möchte und was eben nicht. So könnte die Entfremdung der Kunden vom Produkt langfristig überwunden werden. Insofern zeigt der Ernährungsreport 2017 der Bundesregierung ${ }^{1}$ einen wichtigen Trend auf, der fast altbacken klingt. Wurden in den 70er und 80er Jahren noch reihenweise Schulküchen u. a. zugunsten der Emanzipation geschlossen, begrüßen heutzutage rund 90\% der Deutschen ein eigenes Schulfach Ernährungslehre, um Kindern und Jugendlichen den Umgang mit Lebensmitteln und den Spaß am Kochen wieder zu ermöglichen. So sind es gerade junge Männer im Alter zwischen 18 und 29 Jahren, die mangels Wissen über Küchenhygiene am häufigsten von Infektionen mit Campylobacter durch nicht durchgegartes Hähnchenfleisch betroffen sind.

Wichtig scheint Entspannung - in jeglicher Hinsicht: Gelassenheit gesundheitlichen Risiken gegenüber im Vertrauen auf sichere Lebensmittel, innere Ruhe und Einkehr bezüglich der immerwährenden persönlichen Gewissensbisse, schon wieder das falsche gegessen zu haben sowie bewusst erlebter Genuss, wenn wir es doch wieder geschafft haben, mit Familie, Freunden oder Arbeitskollegen eine gemeinsame, womöglich sogar selbst zubereitete Mahlzeit zu zelebrieren. Na dann guten Appetit!

\footnotetext{
${ }^{1}$ http://www.bmel.de/SharedDocs/Downloads/Broschueren/ Ernaehrungsreport2017.pdf?_blob=publicationFile Zugriff am 17.01.2017.
} 